\title{
New Criterion for Aircraft Multiaxial Fatigue Analysis
}

\author{
Tetiana M aslak ${ }^{1,}{ }^{*}$, Mikhail Karuskevich ${ }^{1}$, and Eukasz Pejkowski ${ }^{2}$ \\ ${ }^{1} \mathrm{~N}$ ational A viation U niversity, A ircraft Design Department, Komarova ave. 1, $03058 \mathrm{~K}$ yiv, Ukraine \\ ${ }^{2}$ University of Science and Technology, Faculty of M echanical Engineering, K aliskiego 7, 85794 \\ Bydoszcz, Poland
}

\begin{abstract}
The complexity of analytical and experimental estimation of aircraft components fatigue life is determined by the irregular character of the load's sequence, a number of stress concentrators, multiaxial stress state. Proposed early multiaxial fatigue criteria are aimed to reduce the complex multi axial loading to an equivalent uniaxial loading. These criteria cover different categories of metals but taking into account the wide variety of constructional materials, modes of loading, environmental conditions, the instrumental structural health monitoring looks a reasonable alternative or at least a strong complement to existing multiaxial fatigue analysis procedures. The new criterion has been proposed as a result of multi-scale levels study of metal surface transformation under fatigue.
\end{abstract}

\section{Introduction}

Damage Tolerance concept currently used in aviation relies on the knowledge of the individual aircraft fatigue damage and the possibility to predict the further process of the fatigue damage accumulation and fracture. The information about the accumulated fatigue damage of the aircraft is valuable also for the implementation of the Limit of Validity (LOV) concept developed for aging aircraft.

Neither analytical calculations nor full-scale tests do not provide with confident data concerning accumulated fatigue damage for individual aircraft in operation. The especially difficult situation with fatigue assessment under the multiaxial loading.

Parts of the plane subjected the variety of operational loads in air and on the ground. As a result of bending and twist, pressurization and accidental loads, the normal and shear stresses arise in phase and out of phase modes, causing fatigue damage of the unpredictable value. Existing methods for multiaxial fatigue assessment do not cover all possible combinations of stresses; thus empirical methods of accumulated fatigue damage assessment combined with adequite nondestructive inspection of metal state are demanded.

\footnotetext{
* Corresponding author: maslakt@yahoo.com
} 


\section{Multiaxial Fatigue Criteria}

Assessment of the accumulated fatigue damage can be carried out by analytical methods, and instrumental diagnostics (direct diagnostic of structure or application of attached and embedded indicators) or by simultaneous complementary application of both.

Like the prediction of fatigue life under the uniaxial fatigue, the analytical methods for multiaxial cyclical loading take into account the mechanical properties of investigated metals, whether these are ductile or plastic materials, first.

Recently developed, accepted, and used in practical procedures analytical multiaxial criteria depending the mechanical properties are divided into stress-based; strain-based; energy-based.

For plastic metals to which the most aviation constructional metals refer, the generally accepted method is based on the Mises criterion for equivalent uniaxial stress.

As there is no exact border between the brittle and plastic metals, the Mises rule has an empirical nature, with inherent errors. Nevertheless, this rule still in use even in aviation where requirements to the accuracy of fatigue life prediction are very strong.

Reviews on the methods for the multiaxial fatigue reveal a variety of contemporary approaches to multiaxial fatigue analysis [1-3], but the practical application of these methods in the aviation industry is rather limited.

Discussed nowadays instrumental methods for multiaxial fatigue analysis are not of the separate "multiaxial" category, these methods like those proposed for uniaxial fatigue are based on the measurements of indirect metal state parameters or accompanied processes, ignoring the special phenomenological features of the multiaxial fatigue damage [4-5].

The discussed method is an extension of the early proposed method for fatigue monitoring of Al-clad aluminum alloy based on the possibility of the quantitative analysis of surface extrusion/intrusion structure.

\section{Extrusion/intrusion structure under fatigue}

Extrusion/intrusion structure observations under fatigue belong to fatigue analysis standards. The first studies of the persistent slip bands were conducted in the early researches of metals deformation and since then are inherent components of fundamental and applied studies.

The process of the extrusion/intrusion structure origin and development reflects the fundamental nature of fatigue damage, and that is why it was proposed to use this phenomenon for practical tasks of the fatigue quantitative assessment in aviation industry.

First, the single crystal indicator attachable to the critical aircraft component was proposed and tested [6], where the density of persistent slip bands indicates the accumulated fatigue damage.

Then it was shown that the extrusion/intrusion structure is visible even on the surface of some industrial materials. The covered by the pure aluminum, so-called Al-clad alloys were studied [7]. The surface of the aluminum cladding layer under fatigue was investigated by the light, scan, transmission, and atomic microscopy [7, 8]. The images of deformation relief, obtained by scan microscopy (fig.1a) and atomic microscopy (fig. 1b) shows 3dimensional nature of the surface pattern.

The evolution of the extrusion/intrusion structure also called deformation relief was found to evolve in the close relation to the number of cycles, stress level, parameters of loading cycle. The intensity of surface damage was assessed as a saturation of the surface with micro plastic deformation signs. 


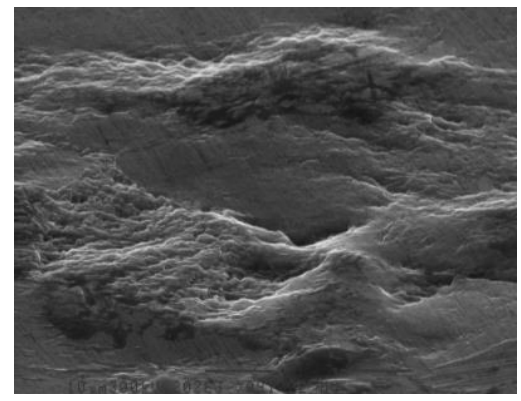

a)

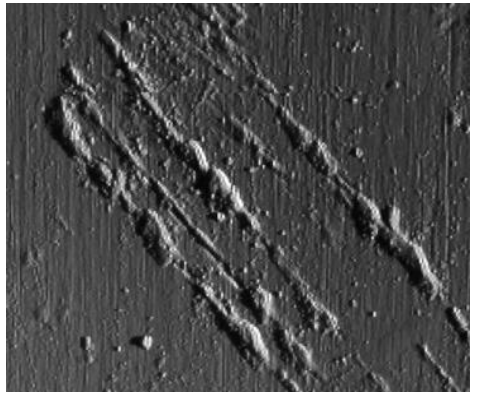

b)

Fig. 1. Deformation relief pattern: a) extrusions/intrusions (2500x); b) persistant slip bands (2000x).

The damage parameter was proposed, calculated as:

$$
D=\frac{S_{\text {def.relief }}}{S_{\text {total }}}
$$

where $S_{\text {def.relief }}$ - surface area with the signs of deformation relief; $S_{\text {total }}$ - total investigated area of the surface.

For the materials which do not exhibit extrusion/intrusion structures under fatigue due to their composition, the application of sensitive fatigue indicator, made of Al-clad alloy attachable to the critical component of the aircraft, for example spar of the wing at the root section has been proved to be the option [10].

The method of fatigue damage estimation by extrusion/intrusion structure reflects two very essential fundamental properties of the metal fatigue: deformation relief forms on the surface revealing the fact that the fatigue damage nucleates mainly at the surface; the analyzed extrusion/intrusion structure being a feature of the micro plastic deformation has a dislocation nature, thus crystallographic orientation of grains has an impact on the process. The influence of the texture rolling requires special attention. It should be mentioned here that all specimens described below had a longitudinal axis along the rolling texture direction.

The results of the deformation relief study under the different modes of uniaxial loading have shown that extrusion/intrusion structure can be considered as a reliable indicator of accumulated fatigue damage. The strong correlations between the intensity of the deformation relief and number of cycles, parameters of loading cycle, level of maximum stress ratio, etc. have been found. Moreover, data expressed as a relation between the consumed life fraction and damage parameter $D$ in the range of stresses closed to the operational level is shown to be described by one generalized curve (fig.2).

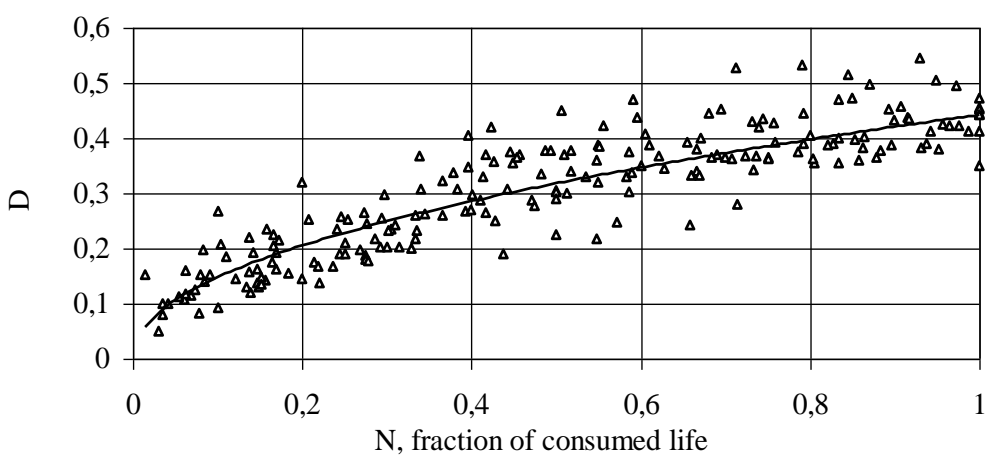

Fig. 2. Damage parameter evolution as a function of fraction of consumed fatigue life for the tests in the range of stresses $\sigma_{\max }=70-120 \mathrm{MPa}, \mathrm{R}=0$. 
These data can be transformed into the diagram for the prediction the remaining fatigue life (fig.3) with regression curve:

$$
N_{\text {remaining }} \%=-226.3 D+119.29
$$

where $D$ - damage parameter. An accuracy of the prediction described by the coefficient of correlation $R=0.89$.

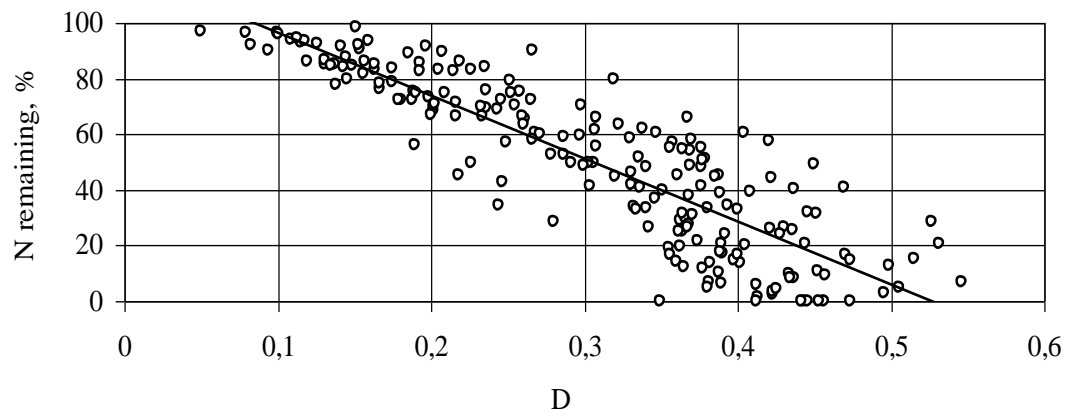

Fig. 3. Generalized relation between the remaining life, $\%$ and damage parameter $D$ for the range of stresses $\sigma_{\max }=70 \mathrm{M} \mathrm{Pa}-120 \mathrm{MPa}, \mathrm{R}=0$.

The typical deformation relief evolution is presented in fig.4a. The deformation relief has a fractal nature $[9,10]$. The "box counting" method was used for the calculation of some types of fractal dimensions, then the dimension $D_{p / s}$ has been found as a more appropriate (fig. 4 b). The fractal dimension $D_{p / s}$ is a characteristic of a clusters relief shape and based on the ratio of the perimeter to their area:

$$
D_{p / s}=\lim _{\delta \rightarrow 0} \frac{\operatorname{LnN}_{p}(\delta)}{\operatorname{LnN}(\delta)},
$$

where $N_{p}(\delta), N_{s}(\delta)$ - number of cells with the size $\delta$ in pixels, which cover the perimeter and area of deformation relief clusters respectively.

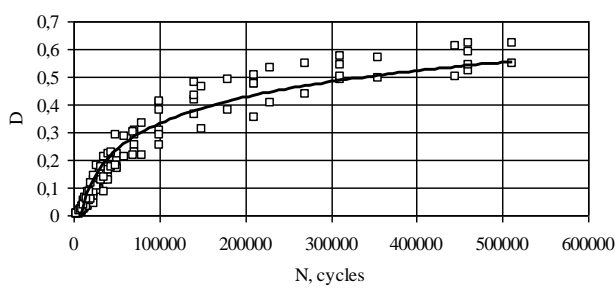

a)

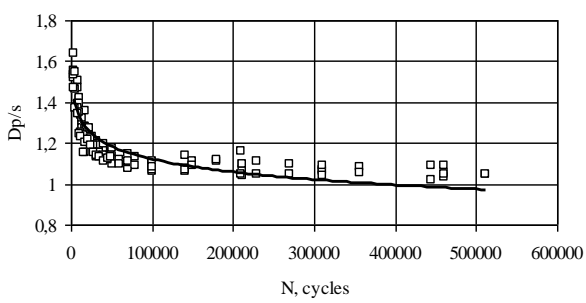

b)

Fig. 4. D eformation relief parameters under the cyclical bending loading at $\sigma \max =173 \mathrm{M} \mathrm{Pa}, R=0$ : a) damage parameter $D$ evolution, b) fractal dimension $D_{p / s}$ evolution.

The application of fractal dimensions of deformation relief clusters in multiple regressions improves the accuracy of the damage assessment.

The multiple regression for the bending loading at $\sigma_{\max }=173 \mathrm{MPa}, \mathrm{R}=0$ has a view:

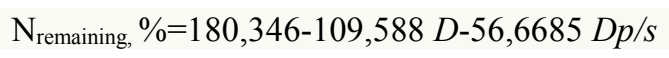

The coefficient of correlation for this equation is $\mathrm{R}=0,84$. 
The procedure of the remaining life prediction can be done by the direct monitoring of alclad alloys and by the monitoring with surface relief fatigue indicators, attached to the critical components of the aircraft primary structure.

The aim of the current investigation has been to prove the sensitivity of the extrusion/intrusion structure to the action of additional components of stress. The proof of this phenomenon allows application of the extrusion/intrusion criteria not only for uniaxial fatigue but for the multiaxial fatigue as well.

The experiments on the alclad aluminum alloy D16AT have been carried out under the standard modes of multiaxial loading.

The first step of the tests has been conducted under the combined action of bending and torsion (Fig.5). The specimens were of $130.0 \mathrm{~mm}$ length, $10.0 \mathrm{~mm}$ width, $1.0 \mathrm{~mm}$ thickness. The drilled hole of $1.0 \mathrm{~mm}$ diameter was a stress concentrator.

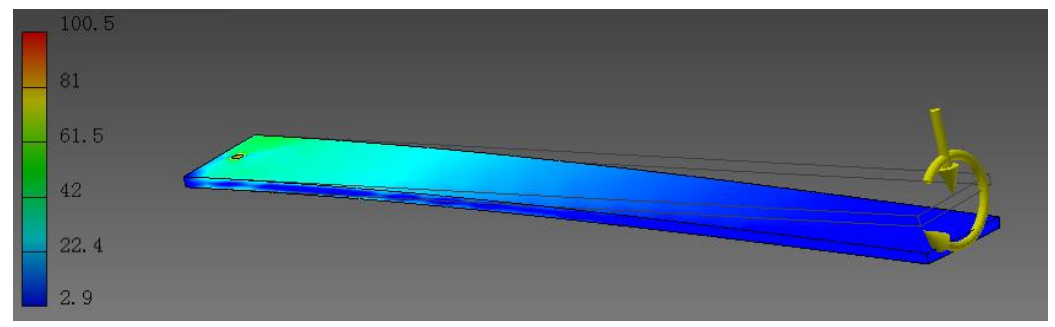

Fig. 5. Finite element simulation of combined loading.

The surface of the specimen subjected to the combined loading (bending and torsion) was compared with those tested under the axial loading (bending, $\sigma_{\max }=100 \mathrm{M} P a, R=0$ ). It was observed the evident difference of the relief saturation rate for these two modes of loading (Fig. 6).

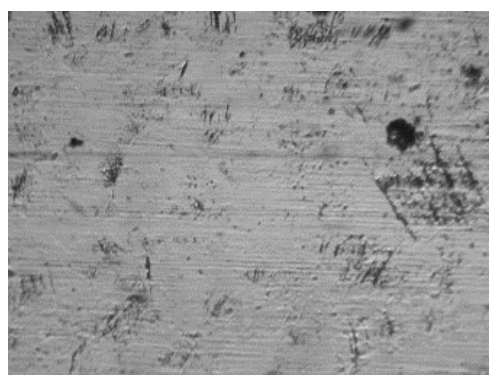

a)

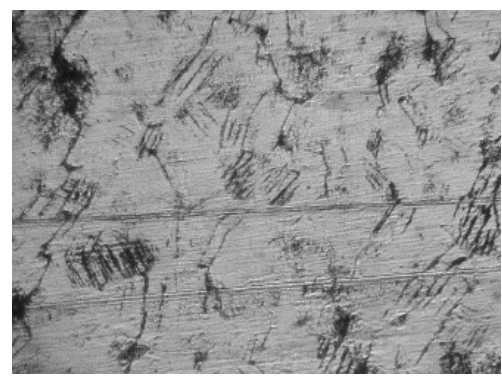

b)

Fig. 6. The deformation relief at $\mathrm{N}=100000$ cycles under: a) uniaxial loading, b) combined loading.

The results obtained at the preliminary stage of the study have proved the sensitivity of the surface extrusion intrusion structures to the action of additional stress components. That is why the research was extended with standard specimens on the machine for the multiaxial loading. Specimens dimensions are given in Fig. 7.

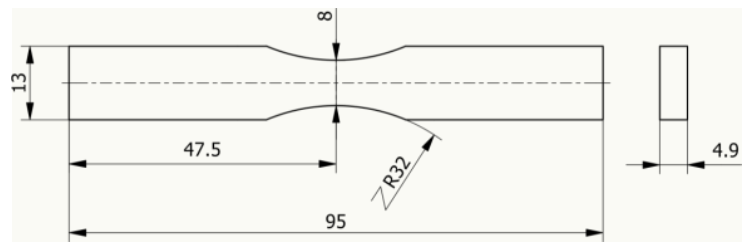

Fig. 7. The geometry of the D16AT specimen. 
For the selection of the loading regimes, the real operational stresses in the aircraft bearing components made of D16AT aluminum alloy have been considered. The reference level of a Huber-M ises equivalent stress equal to $150 \mathrm{M} \mathrm{Pa}$ was selected. The experiments were conducted on the Instron 8874 Axial-Torsional Servo-Hydraulic Fatigue Testing System.

Optical investigations of the surface pattern were conducted by light metallographic microscope Delta Optical IM-100. In addition to the light microscopy analysis, the scan microscopy by the Selmi REM $106 \mathrm{U}$ has been conducted [11].

For the biaxial loading was taken the regime of the proportional loading (combined tension-compression and torsion) was: $\sigma_{a}=106,06 \mathrm{MPa} ; \tau_{a}=61,23 \mathrm{MPa}, \mathrm{R}=-1$. For the uniaxial loading (tension-compression), the parameters of the loading were: $\sigma_{a}=106,06 \mathrm{MPa} ; \tau_{a}=0 \mathrm{MPa}, \mathrm{R}=-1$.

The images of the surface relief after 2000,7000, 50000 cycles for two modes of loading are shown in Fig. 8.

As it seen in Fig. 8 the difference of the deformation relief is apparent: this comprises the different intensity of the relief, and what is of great interest the appearance of additional slip directions in some grains when the loading was the multiaxial. The sensitivity of the deformation relief saturation to the mode of loading is confirmed by the correspondent relations of damage parameter $D$ (indicates the intensity of the relief, see papers [7-10]) and a number of applied cycles of loading (Fig. 9).

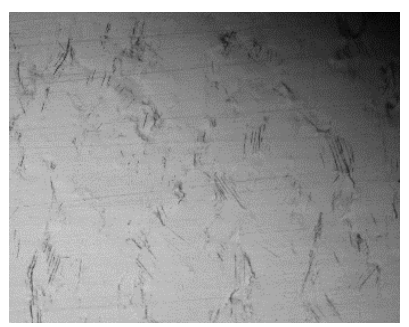

$\mathrm{N}=2000$ cycles

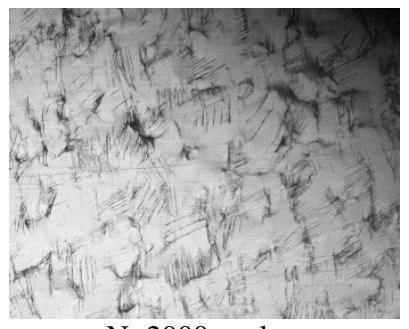

$\mathrm{N}=2000$ cycles

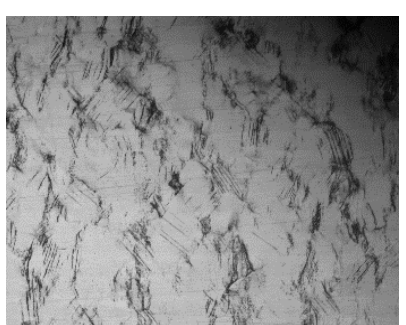

$N=7000$ cycles

a)

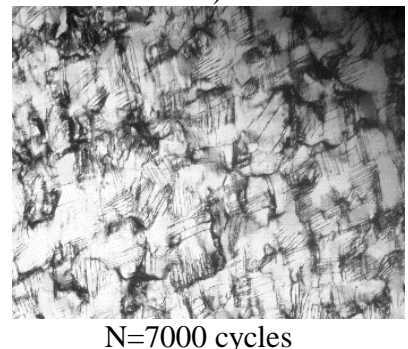

b)
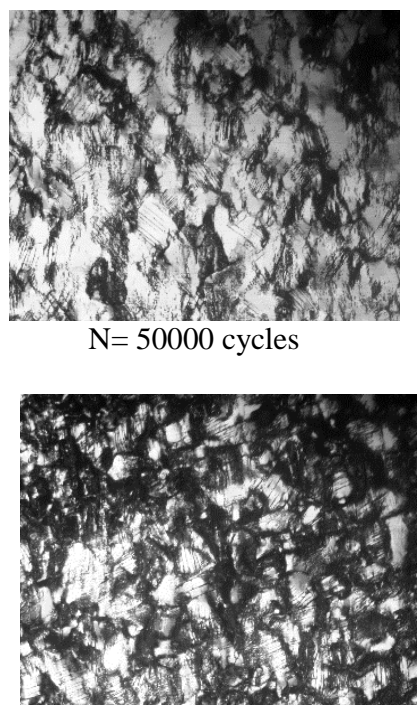

$\mathrm{N}=50000$ cycles

Fig. 8. Evolution of the deformation relief under the uniaxial (a), and biaxial (b) loading.

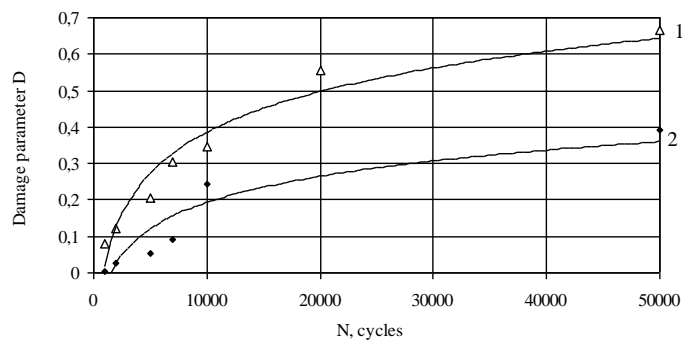

Fig. 9. The deformation relief evolution under the biaxial loading (1) and uniaxial loading (2). 
As it is seen from the graph (fig.9) the initial stage of the relief intensity versus number of cycles relationship both for biaxial and uniaxial loading have monotonic character, so the damage parameter $D$ may be considered as a diagnostic parameter for biaxial loading.

Results of the relief evolution study under the in-phase (proportional loading, curve 1 at fig. 9 and fig. 10) and out-of-phase fatigue loading (fig.10, curve 2), performed in the frame of the same research program presented in the fig. 10. For in-phase and out-of-phase loadings, the shear to normal stress ratio was $\lambda=\tau_{x y} / \sigma_{x}=\sqrt{ } 3$.

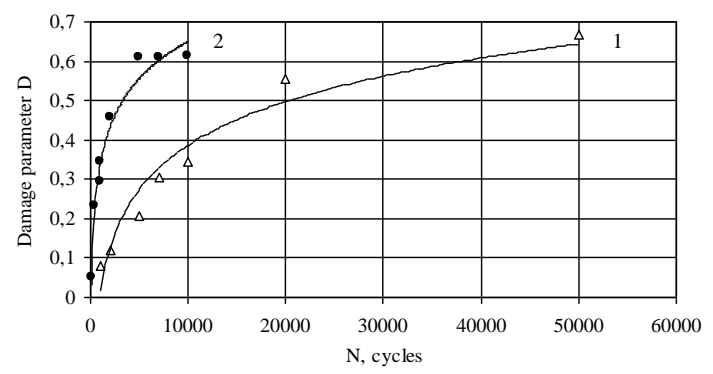

Fig. 10. Comparison of the initial stages of the relief intensity versus number of cycles relationships for biaxial loading: 1-out-of-phase loading; 2-in-phase loading.

The obtained results reveal sensitivity of the extrusion/intrusion structure to the mode of loading: the rate of the damage under the out-of-phase loading bigger than the damage under the in-phase cyclical loading.

The further activity will be directed on the extension the range of loading regimes, the establishment of the relationship between the deformation relief parameters with a remaining life of components and finally, the formalization of the equivalent surface damage concept, which will be based on the comparing the multiaxial relief parameters with relief under the uniaxial cyclical loading.

\section{Conclusions}

The proposed new criterion for aircraft multiaxial fatigue analysis is an extension of the criterion for uniaxial loading statistically validated by the numerical tests.

Extrusion/intrusion criterion reflects the dislocation nature of the fatigue damage as well as its localization in the surface layer of the cyclically deformed metal that is why this approach to the fatigue analysis is considered as a prospective and grounded.

The action of multiple stress components increases the number of actual slip systems and leads to the appearance of new slip tracks on the surface.

It can be stated that extrusion/intrusion structure under multiaxial fatigue is sensitive to the multiaxial mode of loading; thus the new criterion reflects the phenomenology of the multiaxial fatigue and can be considered as a reliable way for fatigue damage quantitative analysis.

\section{References}

1. $Y$ ing- $Y$ u W ang, W ei-Xing $Y$ ao, Int J Fatigue 26, 17-25(2004)

2. A. Karolczuk, E. M acha, Engineering Fracture M echanics 75, 389-403 (2008)

3. Y . Jiang, O. Hertel, M.V ormwald, Int J Fatigue 29 1490-1502 (2007)

4. H. Sohn, C.R. Farrar, F.M. Hemez. Los Alamos National Laboratory Report, LA13976-MS, 311 (2003) 
5. C. B oller, Smart M ater Struct 10, $432-440$ (2001)

6. E.E. Zasimchuk, M.V. Karuskevich, A.I. Radchenko, Fatigue Fract. Eng M ater Struct 15, 1281-1283 (1993)

7. M. Karuskevich, 0. Karuskevich, T. Maslak, S. Schepak, Int J Fatigue 39, 116-121 (2012)

8. T. M aslak, Science-B ased Technologies 2, 140-143 (2013)

9. M. Karuskevych, I. Zhuravel', T. M aslak, M aterials Science 47, 621-626 (2012)

10. M.K aruskevich, S. Ignatovich, M.Petrasek, T.M aslak A dvances in military technology 8, 83-91 (2013)

11. Ł. Pejkowski, M. Karuskevich, T.M aslak, Fatigue Fract Eng M ater Struct 42, 23152324 (2009) 